\title{
Parallelisation of the Petri Net Unfolding Algorithm
}

\author{
Keijo Heljanko $^{1}$, Victor Khomenko ${ }^{2}$, and Maciej Koutny ${ }^{2}$ \\ 1 Laboratory for Theoretical Computer Science, \\ Helsinki University of Technology \\ FIN-02015 HUT, Finland \\ Keijo.Heljanko@hut.fi \\ 2 Department of Computing Science, University of Newcastle \\ Newcastle upon Tyne NE1 7RU, U.K. \\ \{Victor.Khomenko, Maciej.Koutny\}@ncl.ac.uk
}

\begin{abstract}
In this paper, we first present theoretical results, helping to understand the unfolding algorithm presented in 67. We then propose a modification of this algorithm, which can be efficiently parallelised and admits a more efficient implementation. Our experiments demonstrate that the degree of parallelism is usually quite high and resulting algorithms potentially can achieve significant speedup comparing with the sequential case.
\end{abstract}

Keywords: Model checking, Petri nets, parallel algorithms, unfolding, causality, concurrency.

\section{Introduction}

A distinctive characteristic of reactive concurrent systems is that their sets of local states have descriptions which are both short and manageable, and the complexity of their behaviour comes from highly complicated interactions with the external environment rather than from complicated data structures and manipulations thereon. One way of coping with this complexity problem is to use formal methods and, especially, computer aided verification tools implementing model checking (2[1]) - a technique in which the verification of a system is carried out using a finite representation of its state space. The main drawback of model checking is that it suffers from the state space explosion problem. That is, even a relatively small system specification can (and often does) yield a very large state space. To help in coping with this, a number of techniques have been proposed, which can roughly be classified as aiming at an implicit compact representation of the full state space of a reactive concurrent system, or at an explicit generation of its reduced (though sufficient for a given verification task) representation. Techniques aimed at reduced representation of state spaces are typically based on the independence (commutativity) of some actions, often relying on the partial order view of concurrent computation. Such a view is the basis for algorithms employing McMillan's (finite prefixes of) Petri net unfoldings ([6, 
17]), where the entire state space of a system is represented implicitly, using an acyclic net to represent system's actions and local states.

In view of the development of fast model checking algorithms employing unfoldings ([10 11/13]), the problem of efficiently building them is becoming increasingly important. Recently, 5/6/7/15/16 addressed this issue - considerably improving the original McMillan's technique — but we feel that generating net unfoldings deserves further investigation.

The contribution of this paper is twofold. First, we present theoretical results, helping to understand the unfolding algorithm presented in [6]7]. Second, we propose a modification of that algorithm, which can be efficiently parallelised. It does not perform any comparisons of configurations except those needed for checking the cut-off criterion, reducing the total number of times two configuration are compared w.r.t. the adequate total order proposed in [6] down to the number of cut-off events in the resulting prefix. This allows to gain certain speedup even in a sequential implementation. Some other optimisations are also mentioned.

Our experiments demonstrate that the degree of parallelism is usually quite high and the resulting algorithms can potentially achieve significant speedup comparing with the sequential case. All proofs can be found in the technical report [12.

\section{Basic Notions}

In this section, we first present basic definitions concerning Petri nets, and then recall (see also [46]7]) notions related to net unfoldings.

Petri nets. A net is a triple $N \stackrel{\text { df }}{=}(P, T, F)$ such that $P$ and $T$ are disjoint sets of respectively places and transitions, and $F \subseteq(P \times T) \cup(T \times P)$ is a flow relation. A marking of $N$ is a multiset $M$ of places, i.e. $M: P \rightarrow \mathbb{N}=\{0,1,2, \ldots\}$. As usual, we will denote $\bullet^{\bullet} \stackrel{\text { df }}{=}\{y \mid(y, z) \in F\}$ and $z^{\bullet} \stackrel{\text { df }}{=}\{y \mid(z, y) \in F\}$, for all $z \in P \cup T$, and $\bullet Z \stackrel{\text { df }}{=} \bigcup_{z \in Z} \cdot z$ and $Z \bullet \stackrel{\text { df }}{=} \bigcup_{z \in Z} z^{\bullet}$, for all $Z \subseteq P \cup T$. We will assume that $\bullet \neq \emptyset \neq t^{\bullet}$, for every $t \in T$.

A net system is a pair $\Sigma \stackrel{\text { df }}{=}\left(N, M_{0}\right)$ comprising a finite net $N=(P, T, F)$ and an initial marking $M_{0}$. A transition $t \in T$ is enabled at a marking $M$ if for every $p \in \bullet^{\bullet} t, M(p) \geq 1$. Such a transition can be executed, leading to a marking $M^{\prime} \stackrel{\text { df }}{=} M-\bullet t+t^{\bullet}$. We denote this by $M[t\rangle M^{\prime}$. The set of reachable markings of $\Sigma$ is the smallest (w.r.t. set inclusion) set $\left[M_{0}\right\rangle$ containing $M_{0}$ and such that if $M \in\left[M_{0}\right\rangle$ and $M[t\rangle M^{\prime}$ (for some $t \in T$ ) then $M^{\prime} \in\left[M_{0}\right\rangle$.

A net system $\Sigma$ is safe if for every reachable marking $M, M(P) \subseteq\{0,1\}$; and bounded if there is $k \in \mathbb{N}$ such that $M(P) \subseteq\{0, \ldots, k\}$, for every reachable marking $M$.

Branching processes. Two nodes (places or transitions), $y$ and $y^{\prime}$, of a net $N=(P, T, F)$ are in conflict, denoted by $y \# y^{\prime}$, if there are distinct transitions 
$t, t^{\prime} \in T$ such that $\bullet \bullet^{\bullet} t^{\prime} \neq \emptyset$ and $(t, y)$ and $\left(t^{\prime}, y^{\prime}\right)$ are in the reflexive transitive closure of the flow relation $F$, denoted by $\preceq$. A node $y$ is in self-conflict if $y \# y$.

An occurrence net is a net $O N \stackrel{\text { df }}{=}(B, E, G)$ where $B$ is the set of conditions (places) and $E$ is the set of events (transitions). It is assumed that: $O N$ is acyclic (i.e. $\preceq$ is a partial order); for every $b \in B,\left.\right|^{\bullet} b \mid \leq 1$; for every $y \in B \cup E, \neg(y \# y)$ and there are finitely many $y^{\prime}$ such that $y^{\prime} \prec y$, where $\prec$ denotes the irreflexive transitive closure of $G$. $\operatorname{Min}(O N)$ will denote the set of minimal elements of $B \cup E$ with respect to $\preceq$. The relation $\prec$ is the causality relation. Two nodes are concurrent, denoted $y$ co $y^{\prime}$, if neither $y \# y^{\prime}$ nor $y \preceq y^{\prime}$ nor $y^{\prime} \preceq y$. We also denote by $x$ co $C$, where $C$ is a set of pairwise concurrent nodes, the fact that a node $x$ is concurrent to each node from $C$. Two events $e$ and $f$ are separated if there is an event $g$ such that $e \prec g \prec f$.

A homomorphism from an occurrence net $O N$ to a net system $\Sigma$ is a mapping $h: B \cup E \rightarrow P \cup T$ such that: $h(B) \subseteq P$ and $h(E) \subseteq T$; for all $e \in E$, the restriction of $h$ to $\bullet e$ is a bijection between $\bullet e$ and $\bullet h(e)$; the restriction of $h$ to $e^{\bullet}$ is a bijection between $e^{\bullet}$ and $h(e)^{\bullet}$; the restriction of $h$ to $\operatorname{Min}(O N)$ is a bijection between $\operatorname{Min}(O N)$ and $M_{0}$; and for all $e, f \in E$, if $\bullet e=\bullet f$ and $h(e)=h(f)$ then $e=f$. If $h(x)=y$ then we will often refer to $x$ as $y$-labelled.

A branching process of $\Sigma([4])$ is a quadruple $\pi \stackrel{\text { df }}{=}(B, E, G, h)$ such that $(B, E, G)$ is an occurrence net and $h$ is a homomorphism from $O N$ to $\Sigma$. A branching process $\pi^{\prime}=\left(B^{\prime}, E^{\prime}, G^{\prime}, h^{\prime}\right)$ of $\Sigma$ is a prefix of a branching process $\pi=(B, E, G, h)$, denoted by $\pi^{\prime} \sqsubseteq \pi$, if $\left(B^{\prime}, E^{\prime}, G^{\prime}\right)$ is a subnet of $(B, E, G)$ such that: if $e \in E^{\prime}$ and $(b, e) \in G$ or $(e, b) \in G$ then $b \in B^{\prime}$; if $b \in B^{\prime}$ and $(e, b) \in G$ then $e \in E^{\prime}$; and $h^{\prime}$ is the restriction of $h$ to $B^{\prime} \cup E^{\prime}$. For each $\Sigma$ there exists a unique (up to isomorphism) maximal (w.r.t. $\sqsubseteq$ ) branching process, called the unfolding of $\Sigma$.

An example (based on the one in [7) of a safe net system and two of its branching processes is shown in Figure1, where the respective homomorphisms $h$ are shown by placing the names of the nodes of the net system in Figure 1(a) inside the conditions and events of the two branching processes. Note that the branching process in Figure 1(b) is a prefix of that in Figure 1(c).

Sometimes it is convenient to start a branching process with a (virtual) initial event $\perp$, which has the postset $\operatorname{Min}(O N)$, empty preset, and no label. We will assume that $h(\perp)^{\bullet}=M_{0}$.

Configurations and cuts. A configuration of an occurrence net $O N$ is a set of events $C$ such that for all $e, f \in C, \neg(e \# f)$ and, for every $e \in C, f \prec e$ implies $f \in C$. The configuration $[e] \stackrel{\text { df }}{=}\{f \mid f \preceq e\}$ is called the local configuration of $e \in E$. A set of conditions $B^{\prime}$ such that for all distinct $b, b^{\prime} \in B^{\prime}, b$ co $b^{\prime}$, is called a co-set. A cut is a maximal (w.r.t. set inclusion) co-set. Every marking reachable from $\operatorname{Min}(O N)$ is a cut.

Let $C$ be a finite configuration of a branching process $\pi$. Then $C u t(C) \stackrel{\text { df }}{=}$ $\left(\operatorname{Min}(O N) \cup C^{\bullet}\right) \backslash{ }^{\bullet} C$ is a cut; moreover, the multiset of places $h(C u t(C))$ is a reachable marking of $\Sigma$, denoted $\operatorname{Mark}(C)$. A marking $M$ of $\Sigma$ is represented in $\pi$ if the latter contains a finite configuration $C$ such that $M=\operatorname{Mark}(C)$. 


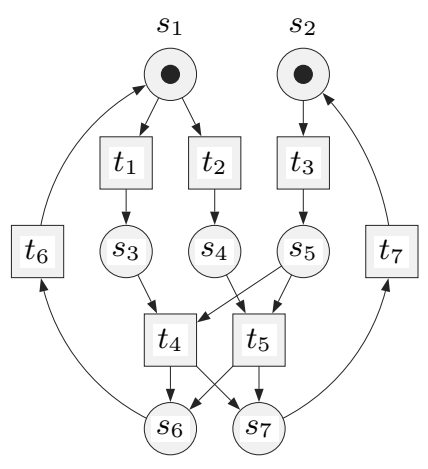

(a)

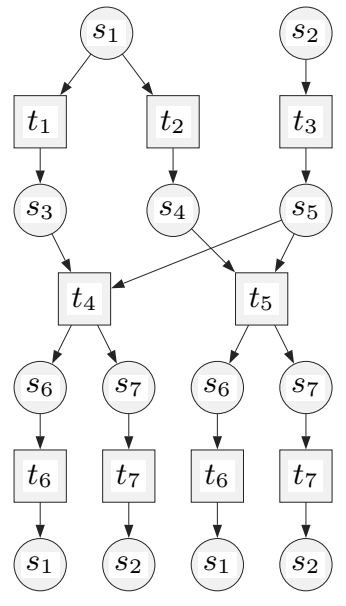

(b)

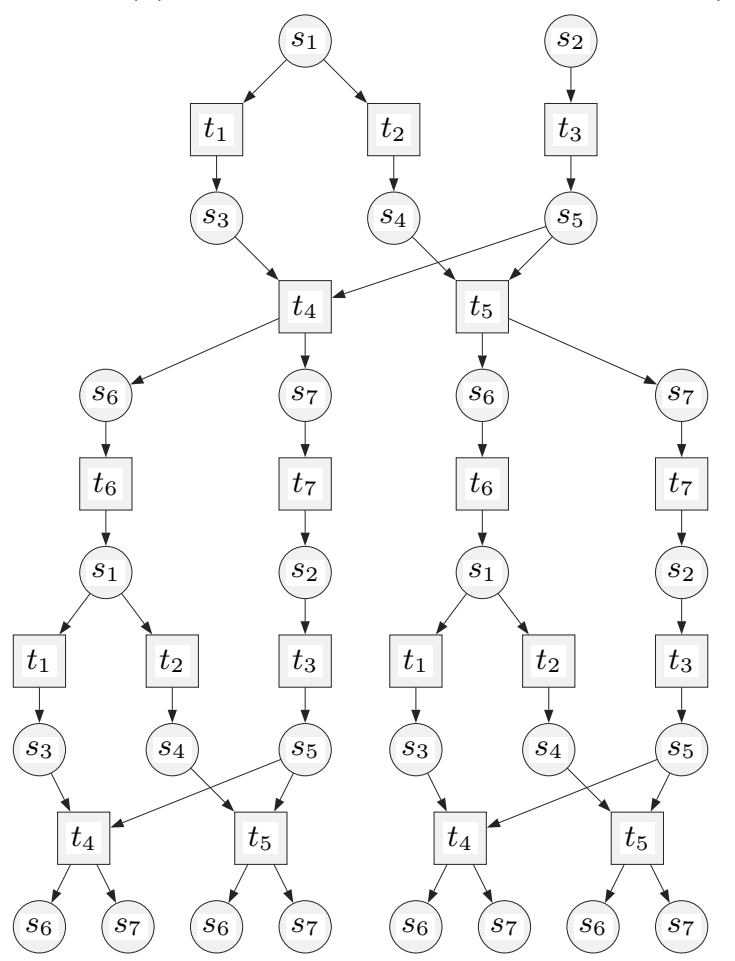

(c)

Fig. 1. A net system (a) and two of its branching processes (b,c). 
Every marking represented in $\pi$ is reachable, and every reachable marking is represented in the unfolding of $\Sigma$.

A branching process $\pi$ of $\Sigma$ is complete if for every reachable marking $M$ of $\Sigma$ : (i) $M$ is represented in $\pi$; and (ii) for every transition $t$ enabled by $M$, there is a finite configuration $C$ and an event $e \notin C$ in $\pi$ such that $M=\operatorname{Mark}(C)$, $h(e)=t$, and $C \cup\{e\}$ is a configuration.

ERV unfolding algorithm. Although, in general, the unfolding of a finite bounded net system $\Sigma$ may be infinite, it is always possible to truncate it and obtain a finite complete prefix, $\operatorname{Pref}_{\Sigma}$. A technique for this, based on choosing an appropriate set $E_{c u t}$ of cut-off events, beyond which the unfolding is not generated, was proposed in [18]. One can show ([69]) that it suffices to designate an event $e$ newly added during the construction of $\operatorname{Pref}_{\Sigma}$ as a cut-off event, if the already built part of a prefix contains a corresponding configuration $C$ without cut-off events, such that $\operatorname{Mark}(C)=\operatorname{Mark}([e])$ and $C \triangleleft[e]$, where $\triangleleft$ is an adequate order, defined in the following way (6/7]).

Definition 1. A strict partial order $\triangleleft$ on the finite configurations of the unfolding of a net system is an adequate order if

$-\triangleleft$ is well-founded,

$-\triangleleft$ refines $\subset$, i.e., $C_{1} \subset C_{2} \Rightarrow C_{1} \triangleleft C_{2}$,

$-\triangleleft$ is preserved by finite extensions, i.e., if $C_{1} \triangleleft C_{2}$ and $\operatorname{Mark}\left(C_{1}\right)=\operatorname{Mark}\left(C_{2}\right)$ then $C_{1} \oplus E \triangleleft C_{2} \oplus I_{C_{1}}^{C_{2}}(E)$ for all finite extensions $C_{1} \oplus E$ of $C_{1}$.

Here $C \oplus E$ denotes the fact that $C \cup E$ is a configuration and $C \cap E=\emptyset$, and $I_{C_{1}}^{C_{2}}$ is a mapping from the finite extensions of $C_{1}$ onto the finite extensions of $C_{2}$, i.e., it maps $C_{1} \oplus E$ onto $C_{2} \oplus I_{C_{1}}^{C_{2}}(E)$ (see [6]7] for details).

We will also write $e \triangleleft f$ whenever $[e] \triangleleft[f]$.

In order to detect cut-off events earlier (and thus decrease the size of the resulting complete prefix), it is advantageous to choose 'dense' (ideally, total) orders, and 677 propose such an order $\triangleleft_{e r v}$ for safe net systems; moreover, it is shown there that if a total order is used then the number of non-cut-off events in the resulting prefix will never exceed the number of reachable markings in the original net system (though usually it is much smaller). The $\triangleleft_{e r v}$ order refines the McMillan's partial adequate order $\left.\triangleleft_{m}([6] 18]\right)$, which is defined as $C_{1} \triangleleft_{m} C_{2} \Longleftrightarrow\left|C_{1}\right|<\left|C_{2}\right|$.

It is often assumed that a corresponding configuration of an event $e$ is the local configuration of some event $f$, which is called a correspondent of a cut-off event $e^{1}$

The unfolding algorithm presented in 5667115]16] (the basic algorithm) is parameterised by an adequate order $\triangleleft$ and can be formulated as shown in Figure2. It is assumed that the function call $\operatorname{PотExT}\left(U n f_{\Sigma}\right)$ finds the set of possible extensions of a branching process $U n f_{\Sigma}$ (see the definition below).

\footnotetext{
1 The more general case of non-local corresponding configurations involves performing a reachability analysis each time when checking whether an event is cut-off, which can be quite time consuming ( 9 ).
} 


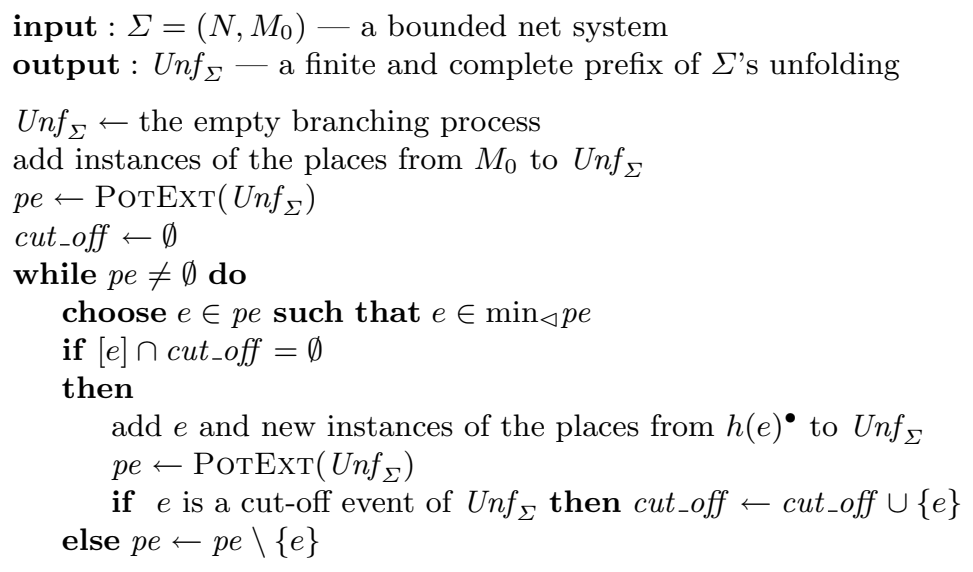

Fig. 2. The unfolding algorithm presented in [6].

Definition 2. Let $\pi$ be a branching process of a net system $\Sigma$, and e be one of its events. A possible extension of $\pi$ is a pair $(t, D)$, where $D$ is a co-set in $\pi$ and $t$ is a transition of $\Sigma$, such that $h(D)=\bullet t$ and $\pi$ contains no $t$-labelled event with the preset $D$. It is a $(\pi, e)$-extension if $e^{\bullet} \cap D \neq \emptyset$, and $e$ and $(t, D)$ are not separated.

Note that in the algorithm, and further in the paper, we do not distinguish between a possible extension $(t, D)$ and a (virtual) $t$-labelled event $e$ with the preset $D$, provided that this does not create an ambiguity. We will also denote by $U n f_{\Sigma}^{S}$, where $S \subseteq \operatorname{PoTExT}\left(U n f_{\Sigma}\right)$, the branching process obtained by adding the events from a set $S$ of possible extensions of $U n f_{\Sigma}$ (together with their postsets) to $U n f_{\Sigma}$.

When $\triangleleft$ is a total order, the algorithm in Figure 2 is deterministic, and thus always yields the same result for a given net system. A surprising fact is that this is also the case for an arbitrary adequate order.

Theorem 1. If $\Sigma$ is a bounded net system then the prefixes produced by two arbitrary runs of the algorithm in Figure 2 are isomorphic.

The above result is also valid in the case when only local corresponding configurations are allowed.

For efficiency reasons, the call to $\operatorname{PoTExT}\left(U n f_{\Sigma}\right)$ in the body of the main loop of the algorithm in Figure 2 can be replaced by a call

$$
\operatorname{UpdatePotExt}\left(p e, U n f_{\Sigma}, e\right),
$$

which finds all $(\pi, e)$-extensions and inserts such events into pe according to the $\triangleleft$ order on their local configurations (see [5[67/16]). 


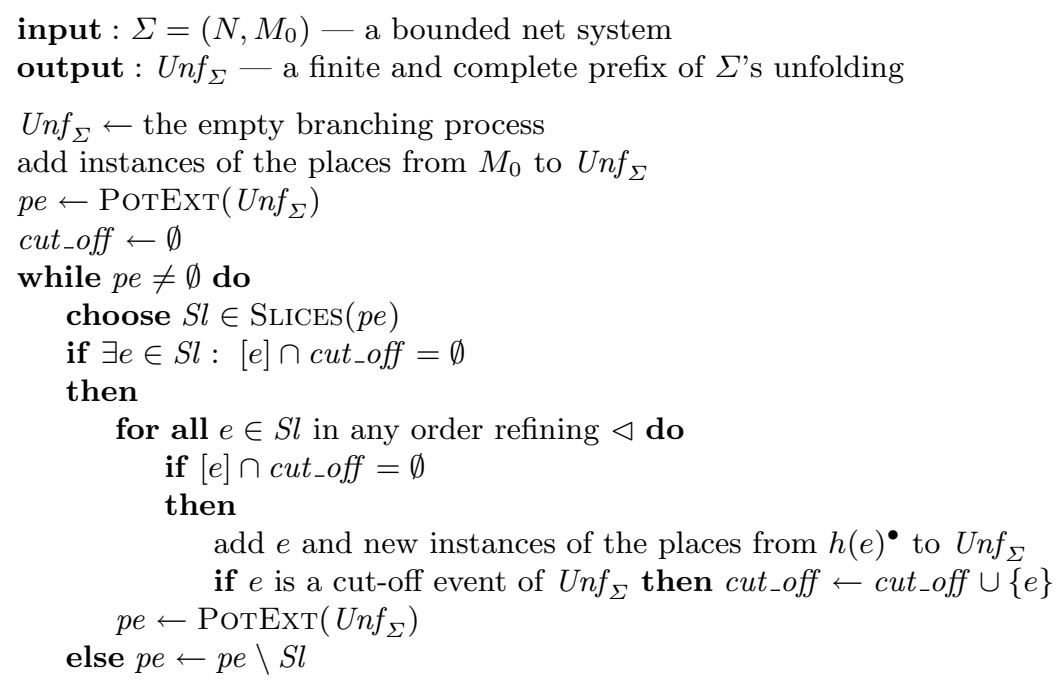

Fig. 3. Unfolding algorithm with slices.

Almost all the steps of the unfolding algorithm can be implemented quite efficiently. The only hard part is computing the set of possible extensions carried out on each iteration of the main loop of the algorithm (a decision version of this problem is, in fact, NP-complete, see [8]10]), and in this paper we will focus our attention on its parallelisation.

\section{Unfolding with Slices}

We now present a general idea behind the parallel unfolding algorithm proposed in this paper. After that we explain how it can be implemented in the case when $\triangleleft$ refines $\triangleleft_{m}$, and discuss further improvements aimed at reducing the amount of performed work.

When looking at the algorithm in Figure 2 one may observe that a possible way of introducing parallelism would be to process several events from pe simultaneously, rather than to insert them one-by-one. This is done in the algorithm in Figure 3 (the slicing algorithm), where the main loop of the algorithm has been modified in the following way. A set of events $S l \in \operatorname{SLICES}(p e)$, called a slice of the current set of possible extensions, is chosen on each iteration and processed as a whole, without taking any other events out from $p e$.

It is assumed that for every $S l \in \operatorname{SLICES}(p e)$ : (i) $S l$ is a non-empty subset of $p e$; and (ii) for every $e \in S l$, if $g$ is an arbitrary event in the unfolding of $\Sigma$ such that $f \prec g$ for some $f \in p e$, or $g \in p e \backslash S l$, then $g \Varangle e$. 
In particular, if $f \in p e$ and $f \triangleleft e$ for some $e \in S l$, then $f \in S l$. The set $\operatorname{SLICES}(p e)$ is chosen so that it is non-empty whenever pe is non-empty. The algorithm in Figure 2 can be seen as a special case of that based on slices, by setting $\operatorname{SLICES}(p e) \stackrel{\text { df }}{=}\left\{\{e\} \mid e \in \min _{\triangleleft} p e\right\}$.

Note that neither any event in $p e \backslash S l$ nor any causal descendant of an event in $p e$ can be less w.r.t. $\triangleleft$ than some event in $S l$. Therefore, if $e \in S l$ is a cut-off event then any of its corresponding configurations is in $U n f_{\Sigma}^{S l}$, where $U n f_{\Sigma}$ is the already built part of the prefix. This essentially means that the events from $S l$ can be inserted into the prefix in any order consistent with $\triangleleft$ (the cut-off events in $S l$ must be identified while doing so). Such a modification of the unfolding algorithm is correct due to the following result.

Lemma 1. If $\Sigma$ is a bounded net system then the algorithm in Figure 3 terminates with a prefix which can be produced by some run of the algorithm in

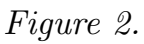

Although the result given by Lemma 1 is sufficient to prove the correctness of our algorithm, a somewhat stronger result, in fact, holds.

Theorem 2. Let Pref $f_{\Sigma}^{\prime}$ and Pref $f_{\Sigma}^{\prime \prime}$ be the prefixes of the unfolding of a bounded net system $\Sigma$, produced by arbitrary runs of the basic and slicing algorithms respectively. Then Pref ${ }_{\Sigma}^{\prime}$ and Pref ${ }_{\Sigma}^{\prime \prime}$ are isomorphic.

This result, together with Theorem 1] suggests that it is possible to define the 'canonical' prefix, which is always generated by the algorithms in Figures 2 and 3 . The theory of such prefixes is developed in [20], where a simpler proof of the correctness of the algorithm in Figure 3 (comparing to the one given in [12]) is provided.

Similarly as for the basic algorithm, the call to PotExT in the body of the main loop of the slicing algorithm can be replaced by a call

$$
\text { UpdatePotExt }\left(p e, U n f_{\Sigma}, S l\right)
$$

which finds all events $f$ such that $f$ is an $\left(U n f_{\Sigma}, e\right)$-extension for some $e \in S l$. The slicing version of the unfolding algorithm provides a basis for subsequent parallelisation, since now possible extensions are derived not from a single event, but rather from a set of events $S l$; it turns out that computing UрdAтеРотЕхт $\left(p e, U n f_{\Sigma}, S l\right)$ can be effectively split into non-overlapping parts and distributed among several processors. Of course, for such scheme to work, we need to ensure that the sets in $\operatorname{SLICES}(p e)$ do satisfy the condition $\left(^{*}\right)$ formulated at the beginning of this section.

\subsection{The Case of an Adequate Order Refining $\triangleleft_{m}$}

When $\triangleleft$ refines $\triangleleft_{m}$ (this is the case for $\triangleleft_{e r v}$ and for most other orders proposed in literature), there is a simple scheme for choosing an appropriate set $\operatorname{SuICES}(p e)$, by setting it to contain all non-empty closed w.r.t. $\triangleleft$ sets of events 
from $p e$ whose local configurations have the minimal size. Then the condition $(*)$ holds. Indeed, suppose that $e \in S l \in \operatorname{SLICES}(p e)$ and $g$ be an event in the unfolding of $\Sigma$. If $f \prec g$ for some $f \in p e$ then it is the case that $|[g]|>|[e]|$. Hence, since $\triangleleft$ refines $\triangleleft_{m}, g \Varangle e$. Moreover, if $g \in p e \backslash S l$ then $g \Varangle e$ as $S l$ is a closed w.r.t. $\triangleleft$ set of events from $p e$.

Notice that in order to achieve better parallelisation, it is advantageous to choose large slices, since this maximizes the number of tasks which can be performed in parallel. Therefore, we can simply choose as a slice the set of all events from $p e$, whose size of the local configuration is minimal (note that this set is closed w.r.t. $\triangleleft$, and, therefore, is in $\operatorname{SLICES}(p e)$ ). With this scheme, we may simply consider $p e$ as a sequence $S l_{1}, S l_{2}, \ldots$ of sets of events such that $S l_{i}$ contains the events whose local configurations have the size $i$ (clearly, in each step of the algorithm there is only a finite number of non-empty $S l_{i}$ 's). Thus inserting an event $e$ into the queue is reduced to adding it into the set $S l_{|[e]|}$, and choosing a slice in the main loop of the algorithm can be replaced by a call Front(pe), returning the first non-empty set $S l_{i}$ in $p e$. Now all the required operations with the queue can be performed without comparisons of configurations at all.

The resulting algorithm is shown in Figure 4. It uses the strategy of finding cut-offs 'in advance' outlined in [15], i.e., it checks the cut-off criterion as soon as a new possible extension is computed. This guarantees that at the beginning of each iteration of the main loop there are no cut-off events in Front(pe), and thus the restriction that the events from $S l$ must be processed in an order consistent with $\triangleleft$ can be safely left out. What is more, this strategy allows one to move the code computing the cut-off criterion into UPDATEPoTExT - the part of the algorithm which is executed in parallel.

When $\triangleleft$ is a total adequate order, each time two configurations are compared w.r.t. $\triangleleft$, one of the events becomes a cut-off event, i.e., the number of the performed comparisons is exactly $\left|E_{\text {cut }}\right|$ (rather than $O(|E| \log |E|$ ) as in former implementations), and the algorithm achieves noticeable speedup even when only one processor is available (see Section 44). One can reduce the number of comparisons even further, using the fact that the local configurations of the events which are already in the prefix are always less than those of newly computed possible extensions. But this would provide almost no speedup, since in this case the sizes of local configurations to be compared always differ, and so the comparisons are fast (we assume that the size of the local configuration is attached to an event).

\subsection{Parallelising the Unfolding Algorithm}

As it was already mentioned, the events in $S l$ can be processed in any order. This leads to a possibility of parallelising the unfolding algorithm when $|S l|>1$. There are only two kinds of dependencies between the events in Sl. First, the cut-off events must be handled properly; this part of the algorithm was explained in the previous section. Second, the $\left(U n f_{\Sigma}, f\right)$-extensions for $f \in S l$ may have in their presets conditions produced by other events from $S l$, inserted into the prefix before $f$. This can be dealt with by inserting all the events from $S l$ into $U n f_{\Sigma}$ 


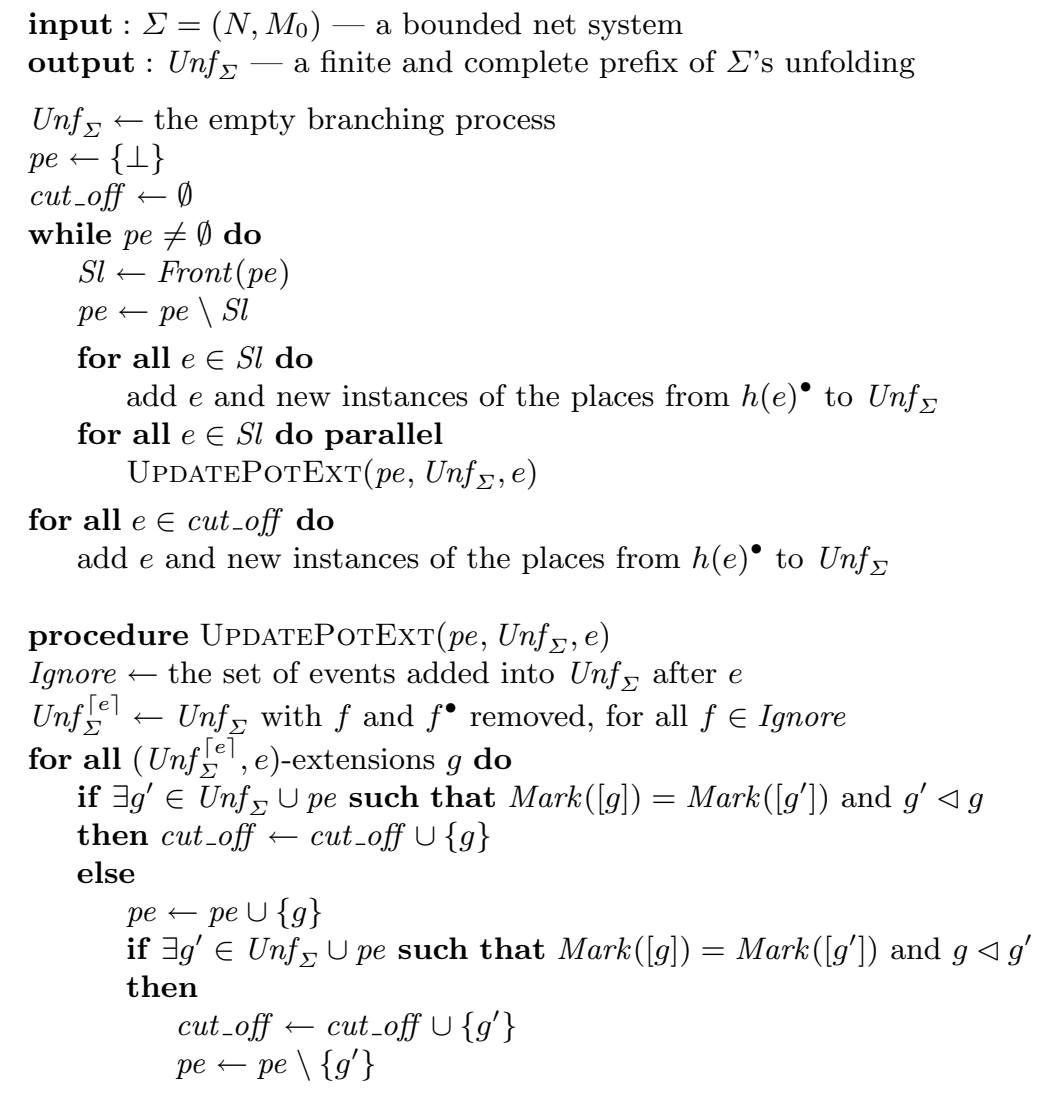

Fig. 4. A parallel algorithm for unfolding Petri nets.

before the loop for computing possible extensions starts, and ignoring some of the inserted events in UPDATEPotExt (see Figure 4).

Since UPDATEPOTExт is the most time-consuming part of the algorithm, this strategy usually provides quite good parallelisation. In the majority of our experiments, there were less than 200 iterations of the main loop, so the time spent on executing the sequential parts of the algorithm was negligible (this fact was confirmed by profiling the program). The first and the last few iterations usually allowed to execute 5-20 UPDATEPотExT's in parallel (which is already enough to provide quite good parallelism for most of the existing shared memory architectures), whereas the middle ones were highly parallel (from several hundreds up to several thousands tasks could potentially be executed in parallel). Thus the scalability of the algorithm is usually very good.

Of course, bad examples do exist, in particular those having 'long and narrow' unfoldings, e.g., the BUF100 net (see Section4). But such examples are very rare 
in practice. Intuitively, they have only a small number of different partial order executions of the same length. This means that they have a very small number of conflicts and a low degree of concurrency (as for the BuF100 example, it has no conflicts at all and allows only few transitions to be executed concurrently). Our experiments show that as soon as the initial conflicts are encountered and added into the prefix being built, the number of events in Front(pe) grows very quickly from step to step.

We implemented our algorithm on a shared memory architecture. It should not be hard to implement it on a distributed memory architecture, e.g., on a network of workstations. In that case, each node keeps a local copy of the built part of the prefix and synchronises it with the master node at the beginning of each iteration of the main loop. The master node is responsible for maintaining the queue of possible extensions, checking the cut-off criterion, and for distributing the work between the slaves; the slaves compute possible extensions and send them to the master.

The idea of slicing the queue also may result in developing a more efficient sequential algorithm. Indeed, we now compute possible extensions for all events in a slice and, therefore, can merge common parts of the work. The technical report [12] describes a simple improvement taking advantage of this idea.

\section{Experimental Results}

We used the sequential unfolding algorithm described in 15,16 as the basis for our parallel implementation and for the comparison (the two implementations share a lot of code, which makes the comparison more fair). In order to experimentally confirm the correctness of the developed parallel implementation, we checked that the produced prefixes are isomorphic to those generated by the sequential version of the algorithm 2 For this, a special utility for 'sorting' prefixes was developed, so that if two prefixes were isomorphic then after 'sorting' they become equal. It works in the following way:

1. Separate cut-off events, pushing them to the end.

2. Sort non-cut-off events according to $\triangleleft_{e r v}$.

3. Separate post-cut-off conditions, pushing them to the end.

4. Sort non-post-cut-off conditions according to the following ordering: $c^{\prime} \lessdot c^{\prime \prime}$ if $e^{\prime} \triangleleft_{e r v} e^{\prime \prime}$, or $e^{\prime}=e^{\prime \prime}$ and $h\left(c^{\prime}\right) \ll h\left(c^{\prime \prime}\right)$, where $\left\{e^{\prime}\right\}={ }^{\bullet} c^{\prime},\left\{e^{\prime \prime}\right\}={ }^{\bullet} c^{\prime \prime}$, and $\ll$ is an arbitrary total order on the places of the original net system (e.g., the size-lexicographical ordering on their names).

Note that $e$ and $e^{\prime}$ are non-cut-off events, and that the of non-cut-off events of the prefix have already been sorted according to $\triangleleft_{e r v}$ by this step.

5. Sort the presets of the events (including the cut-offs) according to $\lessdot$.

6. Sort the cut-off events according to the following ordering: $e^{\prime} \lessdot^{\prime \prime}$ if ${ }^{\prime} e^{\prime} \lessdot_{s l} \bullet e^{\prime \prime}$, or $\bullet e^{\prime}=\bullet e^{\prime \prime}$ and $h\left(e^{\prime}\right) \ll h\left(e^{\prime \prime}\right)$, where $\lessdot_{s l}$ is the size-lexicographical order,

${ }^{2}$ Note that due to Theorem 1 two algorithms using the same adequate order produce isomorphic prefixes (provided that the implementations are correct). See also 20 . 
built upon $\lessdot$, and $\ll$ is an arbitrary total order on the set of the transitions of the original net system (e.g., the size-lexicographical ordering on their names).

Note that the conditions which can appear in the presets of the events have already been sorted by this step.

7. Sort post-cut-off conditions according to $\lessdot$. Note that all events have already been sorted by this step.

8. Sort the postsets of the events (including the cut-offs) according to the $\lessdot$ ordering.

Note that all conditions have already been sorted by this step.

This is an enhanced version of the approach described in [15[16], the only difference is that we can no longer assume that the non-cut-off events in prefixes produced by our algorithm are sorted according to $\triangleleft_{e r v}$, and therefore have to explicitly sort them (step 2).

Test cases. The popular set of benchmark examples, collected by J.C. Corbett ([3]), K. McMillan, S. Melzer, and S. Römer was attempted (this set was also used in [5910111315[1619]). Also we used the $\operatorname{RND}(m, n), \operatorname{SPA}(n)$, and $\operatorname{SPA}(m, n)$ series described in [15]16]. The experiments were conducted on a workstation with four Pentium ${ }^{T M}$ III/500MHz processors and 512M RAM. The parallel algorithm was implemented using Posix threads.

The results of our experiments are summarised in table 1 The meanings of the columns are as follows (from left to right): the name of the problem; the number of places and transitions in the original net; the number of conditions, events and cut-off events in the built complete prefix; the time spent by the sequential unfolder described in 1516; the time spent by the parallel unfolder with different number $N$ of working threads; the average/maximal size of a slice (this characterises the number of independent tasks which may be performed in parallel on each iteration of the main loop). Although, due to the limited number of processors, we could not exploit all the arising parallelism in our experiments, this data shows the potential scalability of the problem.

It is interesting to note that the new algorithm with only one working thread $(N=1)$ works faster than the sequential unfolder described in [15,16]. This is so because it performs much less comparisons of configurations (see Section 3.1) and due to the improvement mentioned at the end of Section 3.2

One can see that our algorithm does not achieve linear speedup. This was a surprising discovery, since the potential parallelism (the last column in the table) is usually very high. Profiling shows that the program spends more than $95 \%$ of time in a function which neither acquires locks, nor performs system calls, so that the contention on locks cannot be the reason for such a slowdown. The only rational explanation we could think of is the bus contention: the mentioned function tries to find co-sets forming presets of possible extensions, exploring

\footnotetext{
${ }^{3}$ We chose only those examples from this set whose unfolding time was large enough to be of some interest.
} 
Table 1. Experimental results.

\begin{tabular}{|c|c|c|c|c|c|c|c|c|c|c|c|}
\hline \multirow[t]{2}{*}{ Problem } & \multicolumn{2}{|c|}{ Net } & \multicolumn{3}{|c|}{ Unfolding } & \multicolumn{5}{|c|}{ Time, $[\mathrm{s}]$} & \multirow[b]{2}{*}{$a / m|S l|$} \\
\hline & $|S|$ & $|T|$ & $|B|$ & $|E|$ & $\left|E_{c u t}\right|$ & ret & & & & $=4$ & \\
\hline $\mathrm{JF}(100)$ & 200 & 101 & 10101 & 5051 & $\overline{11}$ & 31 & 18 & 13 & 13 & 13 & $1.94 /$ \\
\hline & 504 & 409 & 276 & 14724 & 752 & 246 & 183 & 110 & 84 & 78 & \\
\hline & 470 & 343 & & 2737 & & & & & & & \\
\hline & 537 & 392 & 465 & 3896 & 64 & 16 & 12 & & & & \\
\hline & 604 & 441 & 316 & 5337 & 81 & 33 & 26 & 14 & 11 & 10 & $00 / 90$ \\
\hline & 671 & 490 & 191 & 7090 & 100 & 61 & 49 & 28 & 21 & 19 & $2 / 110$ \\
\hline & 738 & 539 & 186 & 9185 & 21 & 105 & 86 & 50 & 39 & 35 & \\
\hline & 57 & 92 & 590 & 7289 & & & 7 & & & 2 & \\
\hline & 66 & 121 & 1558 & 37272 & & 286 & 211 & 126 & 97 & 90 & \\
\hline & 736 & 1939 & 354 & 16935 & 37 & 73 & 42 & 25 & 19 & 17 & \\
\hline & 176 & 529 & 8085 & 89046 & & 2820 & 1609 & 975 & 761 & 714 & $1224 / 3918$ \\
\hline & 53 & 99 & & 18563 & & 30 & 15 & 9 & 7 & & \\
\hline & 258 & 465 & & & & 19 & 11 & 6 & & & \\
\hline & 428 & 841 & 0527 & 50265 & & 884 & 553 & 334 & 259 & 243 & \\
\hline & 1428 & 2705 & 864 & 9933 & & 30 & 18 & 11 & 7 & & $/ 720$ \\
\hline & 129 & 133 & 941 & 6968 & & 10 & 7 & 4 & 3 & 2 & \\
\hline & & 74 & & 67954 & & 935 & 806 & 485 & 379 & 354 & \\
\hline & 22 & 72 & & & & & 4 & 2 & 1 & & \\
\hline & & & & 46902 & & & 339 & 205 & 159 & 150 & \\
\hline & 18 & & & & & & 25 & 15 & 11 & & \\
\hline & 63 & & & 9177 & & 15 & 6 & 3 & & & \\
\hline & 106 & 70 & 138 & 15401 & & 79 & 62 & 36 & 27 & 24 & $/ 369$ \\
\hline & 70 & & & 094 & & & 471 & 284 & 225 & 215 & \\
\hline & 7 & & & & & & 67 & 45 & 274 & 259 & \\
\hline & 8 & & & & & & 4 & 3 & 29 & & \\
\hline & 8 & 5 & & 284 & & & 22 & 03 & 404 & & \\
\hline & 9 & & & 069 & & & & 584 & 469 & 448 & \\
\hline & 4 & & & & & & & & 61 & & \\
\hline & 5 & & & & & & & 140 & 108 & & \\
\hline & & & & & & & & 216 & 168 & & \\
\hline & & & & & & & & 6 & 42 & & \\
\hline & & & & & & & 681 & 411 & 327 & 312 & \\
\hline & & & & & & 7 & 17 & & 6 & & \\
\hline & 45 & & & & & 70 & 28 & 74 & 56 & 49 & \\
\hline & 6( & & & & & & 77 & 162 & 128 & 117 & \\
\hline & & & & & & & 480 & 286 & 228 & 214 & \\
\hline & & & & & & & 42 & & & & \\
\hline & 6 & & & & & & 203 & 118 & 90 & 82 & \\
\hline & & & & & & & 456 & 271 & 213 & 201 & \\
\hline & & & & & & 81 & 48 & 28 & 21 & 19 & $4 / 784$ \\
\hline & & & & 76181 & 45774 & 05 & 603 & 362 & 280 & 264 & $633 / 2612$ \\
\hline & & & & 320582 & 209449 & 13512 & 8066 & 4854 & 3750 & 3537 & \\
\hline & & & & & & & 4 & 2 & 0 & & \\
\hline & & & & & & & 872 & 524 & 406 & 382 & \\
\hline & & & & & & & 4 & 2 & 2 & & \\
\hline & & 65 & 1142214 & 398850 & 256600 & 22011 & 13565 & 8171 & 6317 & 5943 & $2903 / 11807$ \\
\hline
\end{tabular}


the build part of the prefix. It is a fairly large pointer-linked structure, and the processors have to intensively access the memory in a quite unsystematic way, so that the processors' caches often have to redirect the access to the RAM. Therefore, the processors are forced to contend for the bus, and the program slows down. Since this explanation might seem superficial, we decided to establish that bus contention does reveal itself in practice, and the following experiment was performed. Several processors intensively read random locations in a large array and performed some fake computation with the fetched values. The total number of fetches was fixed and evenly distributed among them. In the absence of bus contention, the time spent by such a program would decrease linearly in the number of used processors, but we observed the degradation of speed similar to that shown by our unfolding algorithm. We hope that future generations of hardware will alleviate this problem, e.g., by increasing the bus frequency or by introducing a separate bus for each processor.

\section{Conclusions}

Experimental results indicate that the algorithm we proposed in this paper can achieve significant speedups, at least in theory. But this is still not enough for practical size problems, because the number of processors in shared memory multiprocessors is usually quite small. Therefore, generating unfoldings is still a bottleneck for the unfolding based verification of Petri nets. Our future research will aim at developing an effective implementation of this algorithm for the distributed-memory or hybrid architecture. Another promising area is the approach allowing non-local correspondent configurations, proposed in [9. It sometimes allows to significantly reduce the size of complete prefixes. We plan to investigate if this idea can be efficiently implemented.

Acknowledgements. This research was supported by an ORS Awards Scheme grant ORS/C20/4 and by an EPSRC grant GR/M99293. The financial support of Academy of Finland (Projects 43963, 47754) and Foundation for Technology (Tekniikan Edistämissäätiö) is also gratefully acknowledged.

\section{References}

1. E. M. Clarke, E. A. Emerson and A. P. Sistla: Automatic Verification of Finite-state Concurrent Systems Using Temporal Logic Specifications. ACM TOPLAS 8 (1986) $244-263$.

2. E. M. Clarke, O. Grumberg, and D. Peled: Model Checking. MIT Press (1999).

3. J. C. Corbett: Evaluating Deadlock Detection Methods. University of Hawaii at Manoa (1994).

4. J. Engelfriet: Branching processes of Petri Nets. Acta Informatica 28 (1991) 575591.

5. J. Esparza and S. Römer: An Unfolding Algorithm for Synchronous Products of Transition Systems. Proc. of CONCUR'99, Springer-Verlag, Lecture Notes in Computer Science 1664 (1999) 2-20. 
6. J. Esparza, S. Römer and W. Vogler: An Improvement of McMillan's Unfolding Algorithm. Proc. of TACAS'96, Margaria T., Steffen B. (Eds.). Springer-Verlag, Lecture Notes in Computer Science 1055 (1996) 87-106.

7. J. Esparza, S. Römer and W. Vogler: An Improvement of McMillan's Unfolding Algorithm. Formal Methods in System Design (2001) to appear.

8. J.Esparza and C.Schröter: Reachability Analysis Using Net Unfoldings. Proc. of Workshop of Concurrency, Specification \& Programming 2000 (CSEYP'2000), H. D. Burkhard, L. Czaja, A. Skowron, and P.Starke, (Eds.). Informatik-Bericht 140, vol. 2. Humboldt-Universitat zu Berlin (2000) 255-270.

9. K. Heljanko: Minimizing Finite Complete Prefixes. Proc. of Workshop Concurrency, Specification and Programming 1999 (CSESP'99), (1999) 83-95.

10. K. Heljanko: Deadlock and Reachability Checking with Finite Complete Prefixes. Technical Report A56, Laboratory for Theoretical Computer Science, Helsinki University of Technology, Espoo, Finland (1999).

11. K. Heljanko: Using Logic Programs with Stable Model Semantics to Solve Deadlock and Reachability Problems for 1-Safe Petri Nets. Fundamentae Informaticae 37(3) (1999) 247-268.

12. K. Heljanko, V. Khomenko and M. Koutny: Parallelisation of the Petri Net Unfolding Algorithm. Technical Report CS-TR-733, Department of Computing Science, University of Newcastle (2001).

13. V. Khomenko and M. Koutny: Verification of Bounded Petri Nets Using Integer Programming. Technical Report CS-TR-711, Department of Computing Science, University of Newcastle (2000).

14. V. Khomenko and M. Koutny: LP Deadlock Checking Using Partial Order Dependencies. Proc. of CONCUR'2000, Palamidessi C. (Ed.). Springer-Verlag, Lecture Notes in Computer Science 1877 (2000) 410-425.

15. V.Khomenko and M.Koutny: An Efficient Algorithm for Unfolding Petri Nets. Technical Report CS-TR-726, Department of Computing Science, University of Newcastle (2001).

16. V. Khomenko and M. Koutny: Towards An Efficient Algorithm for Unfolding Petri Nets. Proc. of CONCUR'2001, Larsen P.G., Nielsen M. (Eds.). Springer-Verlag, Lecture Notes in Computer Science 2154 (2001) 366-380.

17. K. L. McMillan: Using Unfoldings to Avoid State Explosion Problem in the Verification of Asynchronous Circuits. Proc. of 4th $C A V$, Springer-Verlag, Lecture Notes in Computer Science 663 (1992) 164-174.

18. K. L. McMillan: Symbolic Model Checking. PhD thesis, CMU-CS-92-131 (1992).

19. S. Melzer and S. Römer: Deadlock Checking Using Net Unfoldings. Proc. of Computer Aided Verification (CAV'97), O. Grumberg (Ed.). Springer-Verlag, Lecture Notes in Computer Science 1254 (1997) 352-363.

20. W. Vogler, V. Khomenko, and M. Koutny: Canonical Prefixes of Petri Net Unfoldings. Technical Report CS-TR-741, Department of Computing Science, University of Newcastle (2001). 